\title{
EXISTENCE AND STABILITY ANALYSIS OF SPIKY SOLUTIONS FOR THE GIERER-MEINHARDT SYSTEM WITH LARGE REACTION RATES
}

\author{
THEODORE KOLOKOLNIKOV, JUNCHENG WEI, AND MATTHIAS WINTER
}

\begin{abstract}
We study the Gierer-Meinhardt system in one dimension in the limit of large reaction rates of the activator. Three solution types are considered: (i) an interior spike; (ii) a boundary spike and (iii) two boundary spikes. It is found that an interior spike is always unstable; a boundary spike is always stable. The two-boundary configuration can be either stable or unstable, depending on the parameters. We fully classify the stability in this case. Numerical simulations are shown in full agreement with analytical results.
\end{abstract}

\section{INTRODUCTION}

In this paper, we study the Gierer-Meinhardt system in the limit of large reaction rates. Let us first put it in the context of Turing's diffusion-driven instability. Since the work of Turing [32] in 1952, many models have been established and investigated to explore the so-called Turing instability [32]. One of the most famous models in biological pattern formation is the Gierer-Meinhardt system [14], [21], [22], which in one dimension can be stated as follows:

$$
\begin{aligned}
& A_{t}=D_{A} \Delta A-A+\frac{A^{p}}{H^{q}}, \quad x \in(-1,1), t>0 \\
& \tau H_{t}=D_{H} \Delta H-H+\frac{A^{m}}{H^{s}} \quad x \in(-1,1), t>0 \\
& A_{x}( \pm 1, t)=H_{x}( \pm 1, t)=0
\end{aligned}
$$

where $(p, q, r, s)$ satisfy

$$
1<\frac{q m}{(s+1)(p-1)}<+\infty, \quad 1<p<+\infty .
$$

In all of the recent mathematical investigations it was always assumed that the activator diffuses much slower than the inhibitor, that is

$$
D_{H} \gg D_{A},
$$

a condition which is related to those required for Turing instability [32]. See Chapter 2 of [23] for a thorough investigation. If the system is studied in a bounded domain, it is further assumed that $D_{A} \ll 1$. In this limit, the GM model becomes weakly coupled in one dimension. Let us summarize some of the recent results about (1) under assumption (2).

1991 Mathematics Subject Classification. Primary 35B40, 35B45; Secondary 35J55, 92C15, 92C40.

Key words and phrases. Stability, Multiple-peaked solutions, Singular perturbations, Turing instability. 
1. Existence of symmetric $N$-peaked steady-state Solutions: First, I. Takagi [31] established the existence of $N$-peaked steady-state solutions with peaks centered at

$$
x_{j}=-1+\frac{2 j-1}{N}, \quad j=1, \ldots, N .
$$

Such solutions are symmetric and they are obtained from a single spike by reflection. Takagi's proof is based on symmetry and the implicit function theorem.

2. Stability of symmetric $N$-peaked solutions: Using matched asymptotic analysis, D. Iron, M. Ward, and J. Wei [18] and, using rigorous proofs, J. Wei and M. Winter [43] studied the stability of symmetric $N$-peaked solutions with $\tau=0$ and the following results are established:

There exists a sequence of numbers $D_{1}>D_{2}>\cdots>D_{N}>\cdots$ (which has been given explicitly) such that if $D_{H}<D_{N}$ the symmetric $N$-peaked solutions are stable, while for $D_{H}>D_{N}$ the symmetric $N$-peaked solutions are unstable.

3. Spike dynamics: Effective equations for the slow motion of spikes in one and two dimensions have been derived in [5], [6], [19], [33], [20]. In two dimensions, the motion of the spike along the boundary has also been described [4], [17].

4. Oscillatory instabilities: When $\tau$ is sufficiently large, the spike solution undergoes a Hopf bifurcation [35], whereby the spike solutions start to oscillate in time.

5. In the shadow system case $\left(D_{H}=\infty\right)$ the existence of single- or $N$-peaked solutions is established in $[1,2,3,8,7,25,26,36,37,45]$ and other papers. In the two-dimensional strong coupling case $\left(D_{H}<\infty\right)$, the existence of 1-peaked solutions is established in [40], and the stability of $N$-peaked solutions is studied in [42]. The two-dimensional weak coupling case $\left(D_{H} \rightarrow \infty\right)$ is investigated in [41] and the existence and stability of multiple-peaked solutions is proved. Instability thresholds similar to the $1 \mathrm{D}$ case are also derived.

6. Existence of asymmetric solutions: By the matched asymptotic analysis approach M. Ward and the second author in [34] and by rigorous proofs the second author and M. Winter in [43] proved the existence of asymmetric $N$-peaked steady-state solutions. Such asymmetric solutions are generated by two types of peaks - called type $\mathbf{A}$ and type $\mathbf{B}$, respectively. Type $\mathbf{A}$ and type $\mathbf{B}$ peaks have different heights. They can be arranged in any given order

АВААВВВ ... АВВВА ... В

to form an $N$-peaked solution. Also the stability of such asymmetric $N$-peaked solutions is studied in [34] and [43], respectively. We remark that symmetric and asymmetric patterns can also be obtained for the Gierer-Meinhardt system on the real line, see [13]. In the real plane, an analogous phenomenon (multi-bump solutions) have also been studied, see for example [9], [10]. 
We now introduce the setting of this paper. In contrast with the above-mentioned works, we do not assume the large diffusivity ratio (2). Instead, we study the the limit of large reaction rates of the activator. More precisely, we assume that

$$
p, m \gg 1 \text { with } O\left(\frac{p}{m}\right)=1
$$

To simplify our analysis, we also set $q=1, s=0, \tau=0$. Moreover rewrite $m=(p-1) r, r=O(1)$. By appropriate scaling, the system becomes

$$
\begin{aligned}
& A_{t}=A_{x x}-A+\frac{A^{p}}{H} ; \quad 0=D H_{x x}-H+A^{(p-1) r}, \quad x \in[-L, L] \\
& A_{x}( \pm L)=0=H_{x}( \pm L) \\
& L, D=O(1) ; \quad p \gg 1 ; \quad r>1 .
\end{aligned}
$$

Hunding and Engelhardt [15] considered first the effect of large reaction rates on Turing's instability for several well-known reaction-diffusion systems (the Sel'kov model, Brusselator, Schnakenberg model, GiererMeinhardt system, Lengyel-Epstein model). By increasing the reaction rate (or the so-called Hill constant $\gamma$ for Hill-type kinetics), they showed, through a linearized stability analysis, that pattern formation by Turing's mechanism is facilitated by increasing cooperativity, even when the ratio of the diffusion rates is close to one.

The case of large reaction rates is well-justified for models of pattern formation induced by gene hierarchy due to their high degree of cooperativity [15]. This process plays a role even for rather primitive animals and plants like flatworm, ciliates, fungi and has been well investigated in Drosophila, where the homeobox genes play a major role [29], [45]. In the latter case key ingredients of the gene hierarchy have been identified such as the maternal gene bicoid, the gap gene hunchback and the primary pair-rule genes, which are expressed in a series of seven equally spaced and precisely phase shifted stripes. The occurence of these stripes can be explained by a Turing mechanism in combination with maternal and gap gene interactions. These mechanisms have been reviewed in [16], [28] and [27].

The cooperativity for homeobox genes is high since they are able to create proteins which bind to several other genes, in this process activating or inhibiting them. Experimentally reaction rates exceeding 8 have been found for several different gene control systems. An explicit example is the pair rule gene hairy which was originally connected to the nervous system but plays a role in the initial body plan of Drosophila as well. This high degree of cooperativity leads to a whole class of control systems with large reaction rates which can explain the emergence of a variety of complex patterns. These large reaction rates further imply that the system can read out and also remember gradients in the positional information which is important since this information is often used repeatedly for example in the anterior-posterior or dorsal-ventral gradients in 
Drosophila. The system also has the property of reacting in an almost on-off manner to very shallow gradients in positional information which plays a major role in controlling the cell cycle governing mitosis since the properties of the system must change qualitatively if its size is increased by a factor 2. Further properties of the resulting nonlinear systems are time oscillations and multi-stability, the latter being important for modelling cell differentiation.

In this paper, we give a first analysis on Turing's nonlinear patterns in the case of large diffusion rate. The model we take is the Gierer-Meinhardt system, though our analysis can well be extended to other reaction-diffusion systems with large nonlinearity (as in [15]).

We now outline the contents of the paper. We begin by constructing the steady-state solution in $\S 2$. Such a solution consists of an inner and outer region, and its construction involves their asymptotic matching. The conclusion is summarized as follows.

Proposition 1. Consider the system

$$
\begin{aligned}
& 0=A_{x x}-A+\frac{A^{p}}{H} ; \quad 0=D H_{x x}-H+A^{(p-1) r}, \quad x \in[-L, L], \\
& A_{x}( \pm L)=0=H_{x}( \pm L) .
\end{aligned}
$$

In the limit

$$
p \rightarrow \infty
$$

let

$$
\alpha:=\frac{1}{p-1} \ll 1
$$

Then (5) admits a solution of the form

$$
\begin{aligned}
& A \sim\left\{\begin{aligned}
\left(\frac{H_{0} \eta}{3 \alpha}\right)^{\alpha} w^{\alpha}\left(\frac{\sqrt{\eta}}{\alpha} x\right), & |x| \ll O(\alpha), \\
\frac{\cosh (|x|-L)}{\cosh (L)}, & |x| \gg O(\alpha),
\end{aligned}\right. \\
& H \sim H_{0} \frac{\cosh \left(\frac{|x|-L}{\sqrt{D}}\right)}{\cosh \left(\frac{L}{\sqrt{D}}\right)},
\end{aligned}
$$

where

$$
\begin{aligned}
H_{0} & =\alpha \eta^{\frac{r-1 / 2}{1-r}}\left[2 \beta^{-1} D^{1 / 2} \tanh \left(\frac{L}{\sqrt{D}}\right)\right]^{1 /(r-1)} \\
\eta & =\tanh ^{2}(L) \\
\beta & =\int_{-\infty}^{\infty} 2^{r} \operatorname{sech}^{2 r}(y / 2) d y
\end{aligned}
$$

and $w$ is the ground state solution to

$$
w_{y y}-w+w^{2}=0, \quad w>0, \quad w_{y}(0)=0, \quad w(y) \sim C e^{-|y|}, \quad|y| \rightarrow \infty
$$




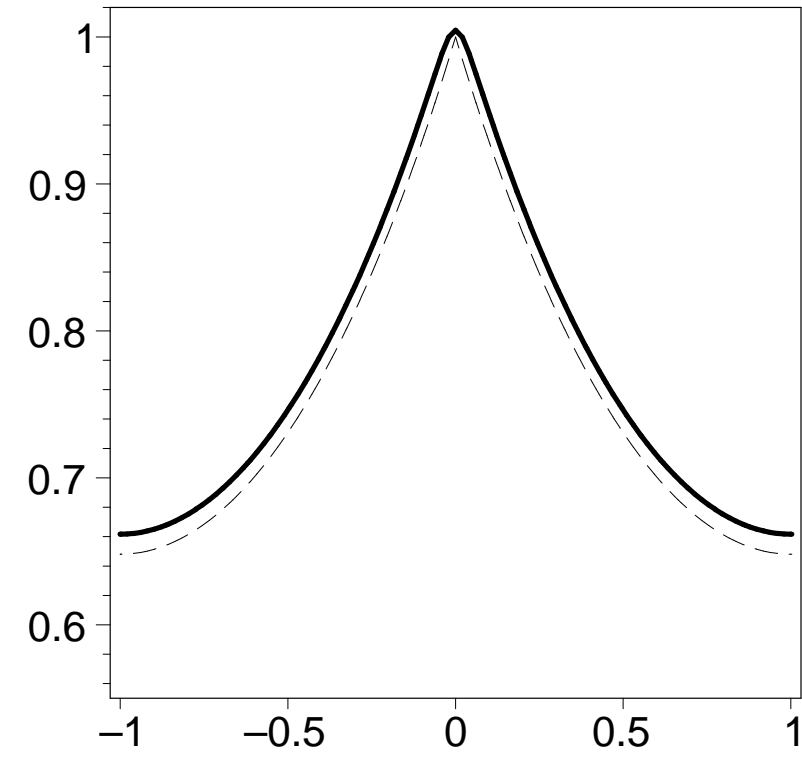

(a)

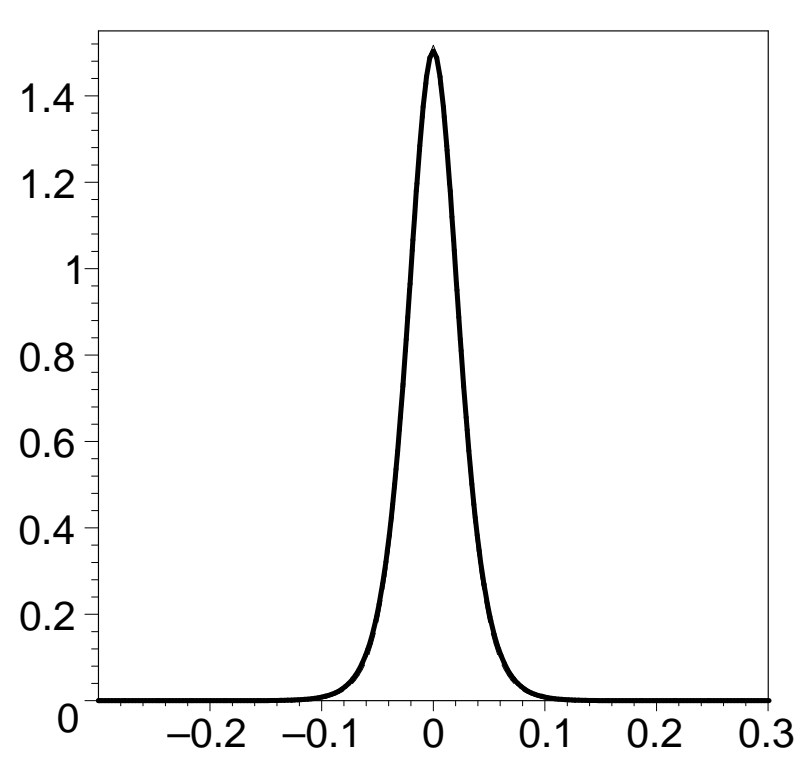

(b)

Figure 1. (a) The plot of the steady state $A(x)$ (solid line) and its outer region asymptotic approximation (dashed line) given by $\cosh (|x|-L) / \cosh (L)$ (b) The plot of $A(x)^{p}$ near the origin (solid line) and its asymptotic approximation given by (6) (dashed line). The parameter values are $p=90, r=2, D=1, L=1$. Note that $A^{p}$ is localized while $A$ is not.

given explicitly by

$$
w(y)=\frac{3}{2} \operatorname{sech}^{2}(y / 2)
$$

The key observation is that unlike in the case of a slowly diffusing activator $\left(D_{A} \ll D_{H}\right)$, the activator $A$ does not look like a spike; nonetheless, its power $A^{p}$ does. This is illustrated in Figure 1, where both $A$ and $A^{p}$ are plotted. Note that $A^{p}$ is localized near the origin while $A$ is not.

A remarkable fact is that in the above proposition, the ratio of the two diffusivities $D$ can be any finite number.

By restricting the domain of solution of Proposition 1 to $[0, L]$, we obtain a boundary spike solution at the left boundary $x=0$. Similarly, by reflecting this boundary spike solution across $x=L$ we get a doubleboundary spike solution. The main result of this paper is the stability analysis for these solutions. We summarize it as follows.

Theorem 2. Suppose $p$ is large enough. A boundary spike is stable. An interior spike is unstable with respect to odd perturbations, and will move towards one of the boundaries. Now consider a double-boundary configuration on the interval $[0,2 L]$, obtained by reflecting the boundary spike on $[0, L]$ along $x=L$. Such a steady state is stable if $D<D_{c}$ and it is unstable if $D>D_{c}$ where $D_{c}$ is the solution to

$$
r \tanh ^{2}\left(\frac{L}{\sqrt{D}}\right)=1
$$




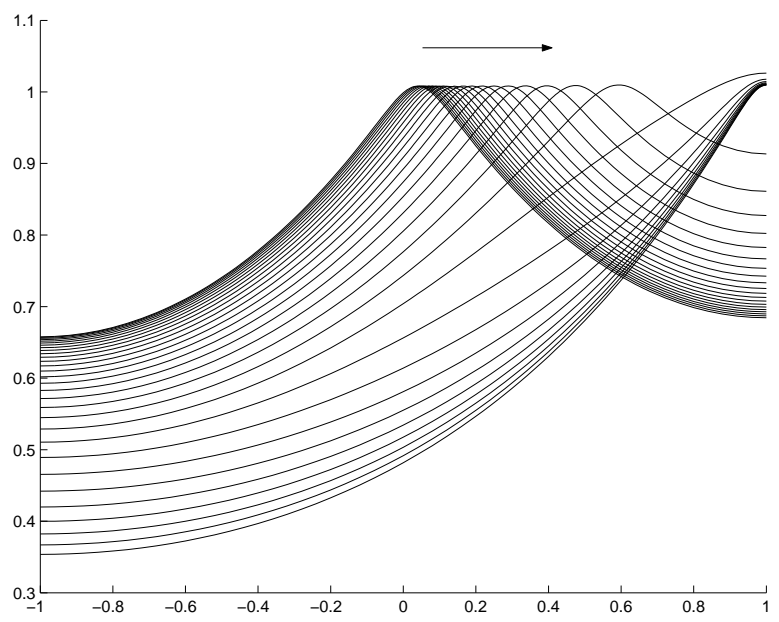

(a)

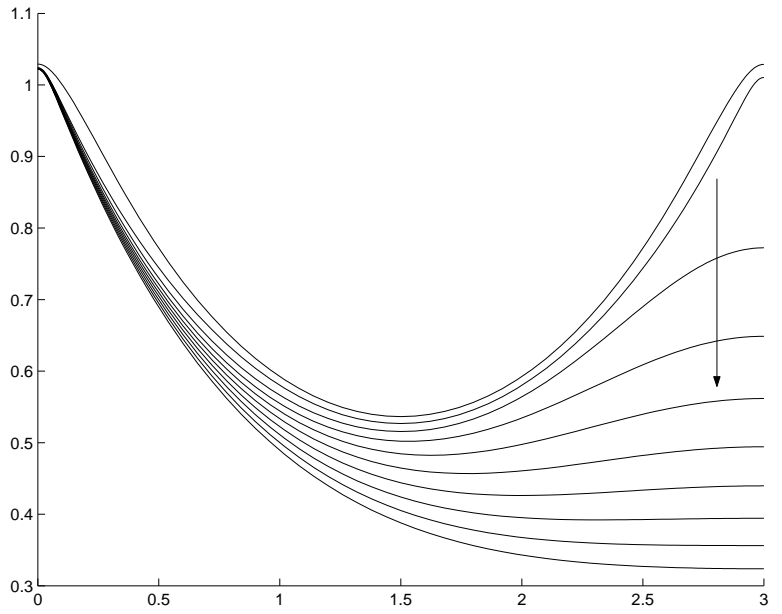

(b)

Figure 2. (a) Motion of the interior spike towards the right boundary. Profiles of $A(x)$ are shown with increments of 0.1 time steps. The parameter values used were $p=50, r=$ $2, D=1, L=1$. The initial condition was taken to be slightly to the right of the center. (b) Competition instability of two boundary spikes. The profiles of $A(x)$ are shown with increments of 0.1 time steps. The spike at the right boundary eventually disappears. Here, $p=50, r=2, D=4, L=1.5$ with $x \in[0,2 L]$.

The two instabilities of Theorem 2 are shown in Figure 2. The instability of the interior spike is due to an unstable "small" eigenvalue whose corresponding eigenfunction is odd. This induces spike motion towards the boundary. On the other hand, the instability of the boundary spike occurs on a much faster timescale, corresponding to a "large" eigenvalue. As a result, one of the two boundary spikes is annihilated.

Note that a multi-spike solution can also be constructed by reflecting an interior spike solution. However, since a single interior spike is unstable, this multi-spike configuration is also automatically unstable so we do not consider it.

We now summarize the contents of the paper. In $\S 2$ we use asymptotic matching to construct the steadystate solution given in Proposition 1. We then formulate the linearized problem in $\S 3$. In $\S 4$ we consider the large eigenvalues. This leads to a nonlocal eigenvalue problem. In Theorem 3 we fully classify its solutions. When $r=2$ we are able to obtain necessary and sufficient conditions for stability. We then study the small eigenvalues in $\S 5$, corresponding to an odd eigenfunction. We show that there is a small positive eigenvalue, so that an interior spike is unstable. We conclude with some open problems in $\S 6$. 


\section{Construction of the steady state}

In this section we construct the steady state usinig asymptotic matching (Proposition 1). As a motivation, note that a solution to the ODE

$$
v_{x x}-v+v^{p}=0
$$

on the whole $\mathbb{R}$ is explicitly given by

$$
v(x)=\left[\left(\frac{p+1}{2}\right) \operatorname{sech}^{2}\left(\frac{p-1}{2} x\right)\right]^{\frac{1}{p-1}} .
$$

This suggests the following change of variables:

$$
A(x)=\left(\frac{u(z)}{\alpha}\right)^{\alpha}, \quad z=\frac{x}{\alpha}
$$

where

$$
\alpha=\frac{1}{p-1} \ll 1
$$

We then obtain the following inner problem:

$$
\begin{aligned}
& 0=u_{z z}-\frac{u_{z}^{2}}{u}+\frac{u^{2}}{H}+\alpha\left(-u+\frac{u_{z}^{2}}{u}\right) \\
& 0=D H_{x x}-H+u^{r} \alpha^{-r} .
\end{aligned}
$$

In the inner region $|x| \ll 1$ we therefore expand

$$
\begin{aligned}
& u(z)=U_{0}(z)+\alpha U_{1}(z)+\cdots \\
& H(z)=H_{0}+\alpha H_{1}(z)+\cdots
\end{aligned}
$$

The leading order equations are

$$
U_{0 z z}-\frac{U_{0 z}^{2}}{U_{0}}+\frac{U_{0}^{2}}{H_{0}}=0, \quad H_{0 z z}=0
$$

so that $H_{0}$ is a constant. A direct verification shows that (12) admits a one-parameter family of solutions given by

$$
U_{0}(z)=\frac{H_{0}}{3} \eta w(\sqrt{\eta} z)
$$

where

$$
w(y)=\frac{3}{2} \operatorname{sech}^{2}(y / 2)
$$

is a solution to (8) and where $\eta$ is an arbitrary parameter that corresponds to a scaling symmetry of (12).

The values for $\eta$ and $H_{0}$ are to be determined shortly.

In the outer region, we have

$$
A_{x x}-A \sim 0 ; \quad A_{x}( \pm L)=0 .
$$


In the inner region we expand in $\alpha$ :

$$
A(z)=\exp \left(\alpha \ln \frac{u(z)}{\alpha}\right)=1+O\left(\alpha \ln \frac{1}{\alpha}\right) .
$$

It follows that $A \rightarrow 1$ as $z \rightarrow \infty$ so that

$$
A \sim \frac{\cosh (L-|x|)}{\cosh L}, \quad|x| \gg O(\alpha) .
$$

Next we perform the matching of the inner and outer solution. For $\alpha \ll|x| \ll 1$ we expand the outer solution in Taylor series to get

$$
\begin{aligned}
A & \sim 1-(\tanh L)|x|+O\left(|x|^{2}\right) \\
& \sim 1-\alpha(\tanh L)|z|+O\left(\alpha^{2}|z|^{2}\right) .
\end{aligned}
$$

On the other hand, note that

$$
w(y) \sim 6 \exp (-|y|), \quad|y| \rightarrow \infty
$$

so that for large $|z|$ we have

$$
\begin{aligned}
A & =\exp \left(\alpha \ln \frac{u}{\alpha}\right) \\
& \sim \exp \left(\alpha \ln \frac{1}{\alpha}\right) \exp \left(\alpha \ln U_{0}\right) \\
& \sim 1+\alpha\left(\ln U_{0}+\ln \frac{1}{\alpha}\right) \\
& \sim 1-\alpha \sqrt{\eta}|z|+O\left(\alpha \ln \frac{1}{\alpha}\right) .
\end{aligned}
$$

Equating the $O(\alpha)$ terms in (14) and (15), we get

$$
\eta=\tanh ^{2} L
$$

To compute $H_{0}$, we note that in the outer region, $A^{(p-1) r} \sim 0$. We therefore write

$$
D H_{x x}-H=-C_{0} \delta(x) ; \quad H_{x}( \pm L)=0
$$

where

$$
\begin{aligned}
C_{0} & =\int_{-\infty}^{\infty} A^{(p-1) r} d x \\
& \sim \frac{\alpha}{\sqrt{\eta}} \int_{-\infty}^{\infty}\left(\frac{\frac{H_{0}}{3} \eta w(y)}{\alpha}\right)^{r} d y \\
& \sim \alpha^{1-r} \eta^{r-1 / 2} H_{0}^{r} \beta
\end{aligned}
$$

and

$$
\beta=\int_{-\infty}^{\infty} 2^{r} \operatorname{sech}^{2 r}(y / 2) d y .
$$

It follows that

$$
H(x)=B \cosh \left(\frac{L-|x|}{\sqrt{D}}\right)
$$


where

$$
\sqrt{D} B \sinh \left(\frac{L}{\sqrt{D}}\right)=\frac{1}{2} C_{0}
$$

so that

$$
\begin{aligned}
& H_{0} \sim H(0)=\frac{1}{2} \frac{C_{0}}{\sqrt{D}} \operatorname{coth}\left(\frac{L}{\sqrt{D}}\right) \\
& H_{0} \sim \alpha \eta^{\frac{r-1 / 2}{1-r}}\left[2 \beta^{-1} D^{1 / 2} \tanh \left(\frac{L}{\sqrt{D}}\right)\right]^{1 /(r-1)} .
\end{aligned}
$$

This completes the derivation of Proposition 1 .

\section{Stability}

We now study the linear stability of the non-homogeneous steady state. Linearize around the steady state as:

$$
\begin{aligned}
& A(x, t)=A(x)+e^{\lambda t} \phi(x) \\
& H(x, t)=H(x)+e^{\lambda t} \psi(x)
\end{aligned}
$$

where $A(x)$ is the solution as given by Proposition 1. We obtain

$$
\begin{aligned}
\lambda \phi & =\phi_{x x}-\phi+p \frac{A^{p-1} \phi}{H}-\frac{A^{p}}{H^{2}} \psi \\
0 & =D \psi_{x x}-\psi+r(p-1) A^{(p-1) r-1} \phi .
\end{aligned}
$$

As before, we make the change of variables given in (11). Since $A \sim 1$ near $x \sim 0$ we have

$$
A^{p}=\frac{u}{\alpha} A \sim \frac{u}{\alpha} .
$$

We obtain

$$
\begin{aligned}
\alpha^{2}(\lambda+1) \phi & \sim \alpha^{2} \phi_{x x}+\frac{u}{H} \phi+\alpha \frac{u}{H} \phi-\alpha \frac{u}{H^{2}} \psi \\
0 & \sim D \psi_{x x}-\psi+r \alpha^{r-1} u^{r} \phi .
\end{aligned}
$$

For an interior spike which is symmetric about the origin, there are two possible eigenfunctions: either odd or even around the origin. Both satisfy Neumann boundary conditions on $[-L, L]$. This yields two separate problems. The even eigenfunction can be restricted to $[0, L]$ and is the same as the eigenfunction for a single boundary spike.

Finally, the double boundary spike on $[0,2 L]$ requires an extra eigenvalue which is odd about $x=L$. This leads to three possible boundary conditions:

- Even eigenfunction for an interior spike on $[-L, L]$ or a boundary spike on $[0, L]$ :

$$
\phi_{x}(0)=0, \phi_{x}(L)=0 ; \psi_{x}(0)=0, \psi_{x}(L)=0 ;
$$




\section{- Interior spike on $[-L, L]$, odd eigenfunction:}

$$
\phi(0)=0, \phi_{x}(L)=0 ; \quad \psi(0)=0, \psi_{x}(L)=0 ;
$$

- Double boundary spike on $[0,2 L]$ :

$$
\phi_{x}(0)=0, \phi(L)=0 ; \psi_{x}(0)=0, \psi(L)=0 .
$$

As will be evident shortly, problems (20) and (22) admit eigenvalues that have $O\left(p^{2}\right)$. We will refer to these as large eigenvalues. These are analyzed in $\S 4$. On the other hand, problem (21) admits an eigenvalue of $O(1)$ which are studied in $\S 5$. We will refer to it as the small eigenvalue.

\section{Large eigenvalues}

We start by analyzing the large eigenvalues. Changing to inner variables, we have

and we obtain

$$
x=\frac{\alpha}{\sqrt{\eta}} y ; \quad u \sim \frac{H_{0}}{3} \eta w(y) ;
$$

$$
\frac{\alpha^{2}}{\eta}(\lambda+1) \phi \sim \phi_{y y}+\frac{1}{3} w \phi-\frac{1}{3} \frac{\psi_{0}}{H_{0}} \alpha
$$

where

$$
\psi_{0}=\psi(0)
$$

and $\psi(0)$ is determined by solving

$$
\begin{gathered}
D \psi_{x x}-\psi \sim C_{1} \delta(x) ; \quad \psi_{x}( \pm L)=0 \\
C_{1}=\int_{-\infty}^{\infty}\left(\frac{u}{\alpha}\right)^{r} \frac{r}{\alpha} \phi d x=\frac{r}{\sqrt{\eta}}\left(\frac{H_{0} \eta}{3 \alpha}\right)^{r} \int_{-\infty}^{\infty} w^{r} \phi d y
\end{gathered}
$$

so that

$$
\psi(x)=-C_{1} G(0)
$$

where

is the Green's function satisfying

$$
G(x)=\frac{\cosh \left(\frac{L-|x|}{\sqrt{D}}\right)}{2 \sqrt{D} \sinh \left(\frac{L}{\sqrt{D}}\right)}
$$

$$
D G_{x x}-G=-\delta(x), \quad G_{x}( \pm L)=0 .
$$

On the other hand, from (16) we have

$$
H_{0}=\frac{\alpha}{\sqrt{\eta}}\left(\frac{H_{0} \eta}{3 \alpha}\right)^{r} \int_{-\infty}^{\infty} w^{r} d y G(0) .
$$

So the boundary conditions (20) lead to following dimensionless nonlocal eigenvalue problem:

$$
\lambda_{0} \phi=\phi_{y y}+\frac{1}{3} w \phi-\frac{r}{3} w \frac{\int_{-\infty}^{\infty} w^{r} \phi d y}{\int_{-\infty}^{\infty} w^{r} d r}, \quad \lambda_{0} \sim \frac{\alpha^{2}}{\eta} \lambda .
$$


For the boundary conditions (22), the only difference is that the boundary conditions in (23) are changed to Dirichlet conditions $\psi( \pm L)=0$. Thus the Green's function now is the one for Dirichlet boundary conditions given by

A similar computation then leads to:

$$
G_{d}(x)=\frac{\sinh \left(\frac{L-|x|}{\sqrt{D}}\right)}{2 \sqrt{D} \cosh \left(\frac{L}{\sqrt{D}}\right)}
$$

$$
\lambda_{0} \phi=\phi_{y y}+\frac{1}{3} w \phi-\frac{r}{3} \tanh ^{2}\left(\frac{L}{\sqrt{D}}\right) w \frac{\int_{-\infty}^{\infty} w^{r} \phi d y}{\int_{-\infty}^{\infty} w^{r} d y}, \quad \lambda_{0} \sim \frac{\alpha^{2}}{\eta} \lambda .
$$

Equations (24), (25) are the starting point of our analysis. Both cases will be covered once we prove the following key theorem.

Theorem 3. Let

$$
L_{0} \phi=\phi_{y y}+\frac{1}{3} w \phi
$$

and consider the nonlocal eigenvalue problem on all of $\mathbb{R}$ :

$$
L_{0} \phi-\gamma w \int_{-\infty}^{\infty} w^{r} \phi d y=\lambda \phi, \quad r \geq 1
$$

where $w$ is given by (8). Let

We have the following:

$$
\gamma_{0}=\frac{1}{3} \frac{1}{\int_{-\infty}^{\infty} w^{r} d y}
$$

(a) If $\gamma<\gamma_{0}$ then (26) has a positive eigenvalue $\lambda>0$.

(b) If $\gamma>\gamma_{0}$ and $r=2$ then $\operatorname{Re}(\lambda)<0$ for all $\lambda$.

Remark: We conjecture that (b) is true for all $r \geq 1$; however we do not know how to prove it in general.

Note that Theorem 3 implies the threshold (10). It also shows that the interior spike is stable with respect to even perturbations.

Before proving Theorem 3, we first summarize the properties of the local operator $L_{0}$. Note that

$$
\begin{aligned}
L_{0} w & =w-\frac{2}{3} w^{2} ; \quad L_{0}^{-1} w=3 \\
\int_{-\infty}^{\infty} w^{2} d y & =6 ; \quad \int_{-\infty}^{\infty} w d y=6 .
\end{aligned}
$$

In addition we have the following characterization of the spectrum of $L_{0}$.

Lemma 4. The even eigenvalues of $L_{0}$ satisfy

$$
\lambda_{1}=\frac{1}{4}, \lambda_{2}<0, \ldots
$$

The eigenfunction corresponding to $\lambda_{1}$ is

$$
\phi_{1}=w^{1 / 2}
$$


Finally, we will need the following key lemma.

Lemma 5. Consider the eigenvalue problem

$$
L_{0} \phi-\frac{1}{18} w \int_{-\infty}^{\infty} w \phi d y=\lambda \phi .
$$

It admits an eigenvalue $\lambda=0$ corresponding to the eigenfunction $\phi=1$. All other eigenvalues are real and strictly negative.

Proof of Lemma 4. We have to solve

$$
\phi_{y y}+\mu w \phi=\gamma^{2} \phi
$$

where, as in [11],

$$
\gamma=\sqrt{\lambda}, \quad \mu=\frac{1}{3}, \quad \phi(y)=w^{\gamma}(y) F(y)
$$

and we take the principal branch of the square root. Then $F$ satisfies

$$
F_{y y}+2 \gamma \frac{w_{y}}{w} F_{y}+\left(\frac{1}{3}-\left(\gamma+\frac{2}{3} \gamma(\gamma-1)\right)\right) w F=0 .
$$

Next we introduce the following new variable

$$
z=\frac{1}{2}\left(1-\frac{w_{y}}{w}\right)
$$

Then

$$
\frac{w_{y}}{w}=1-2 z, \quad w=6 z(1-z), \quad \frac{d z}{d x}=z(1-z) .
$$

This gives the following equation for $F$ as a function of $z$ :

$$
z(1-z) F^{\prime \prime}+(c-(a+b+1) z) F^{\prime}-a b F=0
$$

where

$$
a+b+1=2+4 \gamma, \quad a b=2\left(2 \gamma(\gamma-1)-3\left(\frac{1}{3}-\gamma\right)\right), \quad c=1+2 \gamma
$$

The solutions to (33) are standard hypergeometric functions. See [30] for more details. Now there are two solutions to $(33)$ :

$$
F(a, b ; c ; z) \quad \text { and } \quad z^{1-c} F(a-c+1, b-c+1 ; 2-c ; z) .
$$

By our construction, $F$ is regular at $z=0$. At $z=1, F(a, b ; c ; z)$ has a singularity

$$
\lim _{z \rightarrow 1}(1-z)^{-(c-a-b)} F(a, b ; c ; z)=\frac{\Gamma(c) \Gamma(a+b-c)}{\Gamma(a) \Gamma(b)},
$$

where $c-a-b=-2 \gamma<0$. Note that since $\gamma=\sqrt{\lambda}$, the real part of $\gamma$ is positive. So a solution that is regular at both $z=0$ and $z=1$ can only exist if $\Gamma(x)$ has a pole at $a$ or $b$, respectively. In other words, we have $a, b=0,-1,-2, \ldots$. 
From (34), we compute that

$$
a=2 \gamma-\alpha \text { or } b=2 \gamma-\alpha
$$

where $\alpha$ satisfies

$$
\alpha^{2}+\alpha-2=0
$$

This implies $\alpha=1$ or $\alpha=-2$. By assumption,

$$
2 \sqrt{\lambda}=\alpha+a>0 .
$$

Hence, we have to choose $\alpha=1, a=0$. This gives

$$
\sqrt{\lambda}=\frac{1}{2}, \text { and, finally, } \lambda=\frac{1}{4} .
$$

The corresponding eigenfunction is $w^{1 / 2}$ since $F(0,0 ;-1, z)=1$. We also see that $\lambda=\frac{1}{4}$ is the only positive eigenvalue.

Proof of Lemma 5. Equation (29) is equivalent to solving

$$
\left(L_{0}-\lambda\right) \phi=w ; \quad \int_{-\infty}^{\infty} w \phi d y=18 .
$$

Therefore we define

$$
f(\lambda) \equiv \int_{-\infty}^{\infty} w\left(L_{0}-\lambda\right)^{-1} w d y
$$

so $\lambda$ then solves the equation

$$
f(\lambda)=18
$$

Since (29) is self-adjoint, all eigenvalues are purely real and it suffices to show that $f(\lambda) \neq 18$ for $\lambda>0$. Note that

$$
L_{0} 1=\frac{1}{3} w
$$

so that

$$
f(0)=18
$$

and therefore $\lambda=0$ is an eigenvalue of (29) corresponding to the eigenfunction $\phi=1$. Next, we compute

$$
f^{\prime}(\lambda)=\int_{-\infty}^{\infty} w\left(L_{0}-\lambda\right)^{-2} w d y=\int_{-\infty}^{\infty}\left[\left(L_{0}-\lambda\right)^{-1} w\right]^{2} d y>0
$$

so that $f(\lambda)$ is an increasing function. Finally, note that the local operator $L_{0}$ admits a single positive eigenvalue $\lambda_{0}=\frac{1}{4}$. This implies that $f(\lambda)$ has a single pole at $\lambda=\frac{1}{4}$ and no other poles along the positive real axis $\lambda>0$. On the other hand, for large values of $\lambda$ we have

$$
f(\lambda) \sim-\frac{1}{\lambda} \int_{-\infty}^{\infty} w^{2} d y \rightarrow 0^{-} \text {as } \lambda \rightarrow+\infty .
$$

To summarize, $f(\lambda)$ has a vertical asymptote at $\lambda=\frac{1}{4} ; f(0)=18, f \rightarrow 0^{-}$as $\lambda \rightarrow \infty$ and $f$ is increasing for $\lambda \neq \frac{1}{4}$. It follows that $f(\lambda) \neq 18$ for all $\lambda>0$ and this proves the lemma. 
Proof of Theorem 3. We start with the proof of (a).

Define a function

$$
f(\lambda) \equiv \int_{-\infty}^{\infty} w^{r}\left(L_{0}-\lambda\right)^{-1} w d y
$$

so that the eigenvalue $\lambda$ solves the equation

$$
f(\lambda)=\frac{1}{\gamma}
$$

By (27), note that

$$
f(0)=\frac{1}{\gamma_{0}}
$$

On the other hand, the local operator $L_{0}$ admits a single positive eigenvalue $\lambda_{0}=\frac{1}{4}$. This implies that $f(\lambda)$ has a single pole at $\lambda=\frac{1}{4}$ so that

$$
f(\lambda) \rightarrow \pm \infty \text { as } \lambda \rightarrow\left(\frac{1}{4}\right)^{-}
$$

and $f(\lambda)$ has no other poles along the positive real axis $\lambda>0$. On the other hand, for large values of $\lambda$ we have

$$
f(\lambda) \sim-\frac{1}{\lambda} \int_{-\infty}^{\infty} w^{r+1} d y \rightarrow 0^{-} \text {as } \lambda \rightarrow+\infty
$$

Therefore

$$
f(\lambda) \rightarrow+\infty \text { as } \lambda \rightarrow\left(\frac{1}{4}\right)^{-}
$$

It follows that (40) has a solution with $\lambda<0 \leq \frac{1}{4}$ whenever $0 \leq \gamma<\gamma_{0}$.

Next we prove part (b). Since the operator (26) is not self-adjoint, the eigenvalues are in general complex. Therefore we write

$$
\begin{aligned}
& \lambda=\lambda_{R}+i \lambda_{I} \\
& \phi=\phi_{R}+i \phi_{I} .
\end{aligned}
$$

When $r=2$, we have

$$
\begin{aligned}
L_{0} \phi_{R}-\gamma w \int_{-\infty}^{\infty} w^{2} \phi_{R} d y & =\lambda_{R} \phi_{R}-\lambda_{I} \phi_{I} \\
L_{0} \phi_{I}-\gamma w \int_{-\infty}^{\infty} w^{2} \phi_{I} d y & =\lambda_{R} \phi_{I}+\lambda_{I} \phi_{R} .
\end{aligned}
$$

Multiply (42) by $\phi_{R}$ and (43) by $\phi_{I}$, then integrate and add to obtain

$$
\int_{-\infty}^{\infty}\left(\phi_{R} L_{0} \phi_{R}+\phi_{I} L_{0} \phi_{I}\right) d y-\gamma A=\lambda_{R} B
$$

where

$$
\begin{aligned}
& A=\int_{-\infty}^{\infty} w \phi_{R} d y \int_{-\infty}^{\infty} w^{2} \phi_{R} d y+\int_{-\infty}^{\infty} w \phi_{I} d y \int_{-\infty}^{\infty} w^{2} \phi_{I} d y \\
& B=\int_{-\infty}^{\infty}\left(\phi_{R}^{2}+\phi_{I}^{2}\right) d y .
\end{aligned}
$$


Multiply (42) and (43) by $w$, then integrate by parts. We have

$$
\begin{gathered}
\int_{-\infty}^{\infty} \phi_{R}\left(w-\frac{2}{3} w^{2}\right) d y-6 \gamma \int_{-\infty}^{\infty} w^{2} \phi_{R} d y=\lambda_{R} \int_{-\infty}^{\infty} \phi_{R} w d y-\lambda_{I} \int_{-\infty}^{\infty} \phi_{I} w d y \\
\int_{-\infty}^{\infty} \phi_{I}\left(w-\frac{2}{3} w^{2}\right) d y-6 \gamma \int_{-\infty}^{\infty} w^{2} \phi_{I} d y=\lambda_{R} \int_{-\infty}^{\infty} \phi_{I} w d y+\lambda_{I} \int_{-\infty}^{\infty} \phi_{R} w d y .
\end{gathered}
$$

Eliminating $\lambda_{I}$ we then obtain

$$
\left(\lambda_{R}-1\right) C+\left(\frac{2}{3}+6 \gamma\right) A=0
$$

where $A$ is given by (45) and

$$
C=\left(\int_{-\infty}^{\infty} w \phi_{R} d y\right)^{2}+\left(\int_{-\infty}^{\infty} w \phi_{I} d y\right)^{2} .
$$

Next we use the following estimate, see Lemma 5:

$$
\int_{-\infty}^{\infty} \phi L_{0} \phi d y \leq \frac{1}{18}\left(\int w \phi d y\right)^{2}
$$

Then (44) becomes

$$
\lambda_{R} B+\gamma A \leq \frac{1}{18} C
$$

Combining with (47) we obtain

$$
\lambda_{R} B-\gamma \frac{\left(\lambda_{R}-1\right)}{\left(\frac{2}{3}+6 \gamma\right)} C-\frac{1}{18} C \leq 0
$$

and so

$$
\lambda_{R}\left[B-\frac{\gamma}{\left(\frac{2}{3}+6 \gamma\right)} C\right] \leq\left[\frac{1}{18}-\frac{\gamma}{\left(\frac{2}{3}+6 \gamma\right)}\right] C
$$

Note that

$$
\begin{aligned}
& \gamma_{0}=\frac{1}{18} \\
& \frac{1}{18} \leq \frac{\gamma}{\left(\frac{2}{3}+6 \gamma\right)}<\frac{1}{6} \text { whenever } \gamma_{0} \leq \gamma<\infty
\end{aligned}
$$

so that

$$
\lambda_{R}\left[B-\frac{\gamma}{\left(\frac{2}{3}+6 \gamma\right)} C\right] \leq 0, \quad \gamma \geq \gamma_{0} .
$$

Now by Cauchy-Schwarz inequality we have

$$
C \leq 6 B \Longleftrightarrow B-\frac{1}{6} C \geq 0 .
$$

Combining (50) and (51) we have

$$
B-\frac{\gamma}{\left(\frac{2}{3}+6 \gamma\right)} C \geq 0
$$

Therefore $\lambda_{R} \leq 0$. Further, if $\lambda_{R}=0$, then from (48) and (49) we have

$$
0 \leq\left[\frac{1}{18}-\frac{\gamma}{\left(\frac{2}{3}+6 \gamma\right)}\right] C \leq 0
$$

this can only happen if $\gamma=\frac{1}{18}=\gamma_{0}$. 


\section{Small eigenvalue}

It remains to study the stability of small eigenvalues. In particular, we prove the following result.

Lemma 6. Consider the eigenvalue problem (19a,19b) with the boundary conditions (21). In the limit $p \rightarrow \infty$, this problem admits a positive eigenvalue $\lambda$ that satisfies

$$
\sqrt{\lambda+1} \tanh L \tanh (L \sqrt{\lambda+1})=1 .
$$

To start with, expand the inner region to two orders for both the eigenfunction and the steady state:

$$
\begin{gathered}
x=\alpha z ; \\
u=U_{0}(z)+\alpha U_{1}(z)+\cdots \quad H=H_{0}+\alpha H_{1}(z)+\cdots \\
\phi=\Phi_{0}(z)+\alpha \Phi_{1}(z)+\cdots \quad \Psi=\Psi_{0}+\cdots
\end{gathered}
$$

The leading order equations are

$$
\Phi_{0 z z}+\frac{U_{0}}{H_{0}} \Phi_{0}=0 ; \quad U_{0 z}-\frac{U_{0 z}^{2}}{U_{0}}+\frac{U_{0}^{2}}{H_{0}}=0 ; \quad H_{0} \equiv \text { const. }
$$

The solution to $\Phi_{0}$ is given by

$$
\Phi_{0}(z)=\frac{U_{0 z}}{U_{0}}
$$

We now formulate a solvability condition with $\Phi_{0}$ as a test function. Multiplying (19a) by $\frac{1}{\alpha} \Phi_{0}\left(\frac{x}{\alpha}\right)$ and integrating on the half-interval $[0, L]$, we have,

$$
\alpha^{2}(\lambda+1) \int_{0}^{L} \phi(x) \Phi_{0}\left(\frac{x}{\alpha}\right) \frac{d x}{\alpha}=\int_{0}^{L}\left(\alpha^{2} \phi_{x x}+\frac{u}{H} \phi+\alpha \frac{u}{H} \phi-\alpha \frac{u}{H^{2}} \psi\right) \Phi_{0} \frac{d x}{\alpha} .
$$

First we estimate the $\operatorname{lhs}(55)$. In the outer region we use $w(y) \sim C \exp (-|y|),|y| \rightarrow \infty$ so that

$$
\Phi_{0} \sim-\sqrt{\eta}, \quad z \gg 1
$$

On the other hand, up to exponentially small terms we have

$$
\phi_{x x} \sim(\lambda+1) \phi, \quad x \gg \alpha ; \quad \phi^{\prime}(L)=0
$$

so that we may write

$$
\phi \sim A_{0} \frac{\cosh (\sqrt{\lambda+1}(x-L))}{\cosh (\sqrt{\lambda+1} L)}
$$

where $A_{0}$ is obtained by matching $\phi$ as $x \rightarrow 0$ to $\Phi_{0}$ as $z \rightarrow \infty$. This yields

$$
A_{0}=-\sqrt{\eta}
$$

Therefore we estimate

$$
\begin{aligned}
\int_{0}^{L} \phi(x) \Phi_{0}\left(\frac{x}{\alpha}\right) d x & \sim \eta \int_{0}^{L} \frac{\cosh (\sqrt{\lambda+1}(x-L))}{\cosh (\sqrt{\lambda+1} L)} d x \\
& \sim \frac{\eta}{\sqrt{\lambda+1}} \tanh (\sqrt{\lambda+1} L)
\end{aligned}
$$


and finally

$$
\operatorname{lhs}(55)=\alpha \eta \sqrt{\lambda+1} \tanh (\sqrt{\lambda+1} L)
$$

Next we must estimate the $\operatorname{rhs}(55)$. Since $u$ decays exponentially as $z \rightarrow \infty$, the inner region provides the dominant contribution there. After changing variables $x=\alpha z$ and expanding, we obtain,

$$
\begin{aligned}
\operatorname{rhs}(55) & =\int_{0}^{\infty} \Phi_{0}\left(\Phi_{0 z z}+\frac{U_{0}}{H_{0}} \Phi_{0}\right) d z+\alpha \int_{0}^{\infty} \Phi_{0}\left(\Phi_{1 z z}+\Phi_{1} \frac{U_{0}}{H_{0}}\right) d z \\
& +\alpha \int_{0}^{\infty} \Phi_{0}^{2}\left(\frac{U_{1}}{H_{0}}-\frac{U_{0} H_{1}}{H_{0}^{2}}\right) d z+\alpha \int_{0}^{\infty} \frac{U_{0} \Phi_{0}^{2}}{H_{0}}-\alpha \int_{0}^{\infty} \frac{U_{0} \Phi_{0}}{H_{0}^{1}} \Psi_{0} d z+O\left(\alpha^{2}\right) .
\end{aligned}
$$

The first term is zero by (53); we write the remaining terms as

$$
r h s(55)=\alpha\left(I_{0}+I_{1}+I_{2}+I_{3}\right)
$$

where

$$
\begin{aligned}
I_{0} & =\int_{0}^{\infty} \Phi_{0}\left(\Phi_{1 z z}+\Phi_{1} \frac{U_{0}}{H_{0}}\right) d z \\
I_{1} & =\int_{0}^{\infty} \Phi_{0}^{2}\left(\frac{U_{1}}{H_{0}}-\frac{U_{0} H_{1}}{H_{0}^{2}}\right) d z \\
I_{2} & =\int \Phi_{0}^{2} \frac{U_{0}}{H_{0}} d z \\
I_{3} & =-\int_{0}^{\infty} \frac{U_{0} \Phi_{0}}{H_{0}^{2}} \Psi_{0} d z .
\end{aligned}
$$

Now define

$$
L_{0} \Phi \equiv \Phi_{z z}+\frac{U_{0}}{H_{0}} \Phi
$$

First, we integrate by parts to obtain

$$
I_{0}=\int_{0}^{\infty} \Phi_{0} L_{0} \Phi_{1}=\left[\Phi_{1 z} \Phi_{0}-\Phi_{1} \Phi_{0 z}\right]_{0}^{\infty}=0
$$

Next, $U_{1}$ satisfies

$$
U_{1 z z}-\frac{2 U_{0 z} U_{1 z}}{U_{0}}+\frac{U_{0 z}^{2}}{U_{0}^{2}} U_{1}+2 \frac{U_{0} U_{1}}{H_{0}}-\frac{U_{0}^{2}}{H_{0}^{2}} H_{1}-U_{0}+\frac{U_{0 z}^{2}}{U_{0}}=0
$$

Now define

$$
\hat{U}_{1} \equiv \frac{U_{1}}{U_{0}}
$$

Then $\hat{U}_{1}$ satisfies

$$
\hat{U}_{1 z z}+\frac{U_{0}}{H_{0}} \hat{U}_{1}-\frac{U_{0} H_{1}}{H_{0}^{2}}-1+\frac{U_{0 z}^{2}}{U_{0}^{2}}=0
$$

Differentiating (58) we obtain

$$
\begin{aligned}
L_{0} \hat{U}_{1 z} & =-\frac{U_{0 z} \hat{U}_{1}}{H_{0}}+\frac{U_{0 z} H_{1}}{H_{0}^{2}}+\frac{U_{0} H_{1 z}}{H_{0}^{2}}-\left(\frac{U_{0 z}^{2}}{U_{0}^{2}}\right)_{z} \\
& =-\Phi_{0} \frac{U_{1}}{H_{0}}+\frac{\Phi_{0} U_{0} H_{1}}{H_{0}^{2}}+\frac{U_{0} H_{1 z}}{H_{0}^{2}}+2 \frac{U_{0 z}}{H_{0}} .
\end{aligned}
$$


Therefore we have

$$
I_{1}=-\int_{0}^{\infty} \Phi_{0} L_{0} \hat{U}_{1 z} d z+\int_{0}^{\infty} \frac{\Phi_{0} U_{0} H_{1 z}}{H_{0}^{2}} d z+2 \int_{0}^{\infty} \frac{\Phi_{0} U_{0 z}}{H_{0}} d z .
$$

Integrating by parts, we get

$$
\int_{0}^{\infty} \Phi_{0} L_{0} \hat{U}_{1 z} d z=\Phi_{0}(\infty) \hat{U}_{1 z z}(\infty)
$$

Note that $U_{0} \rightarrow 0, \quad \Phi_{0} \rightarrow-\sqrt{\eta}$ as $z \rightarrow \infty$ and using (58) we obtain

$$
\hat{U}_{1 z z}(\infty)=1-\eta
$$

so that

$$
\int_{0}^{\infty} \Phi_{0} L_{0} \hat{U}_{1 z} d z=-\sqrt{\eta}+\eta^{3 / 2}
$$

Next we compute

Note that $H$ satisfies

$$
\int_{0}^{\infty} \frac{\Phi_{0} U_{0} H_{1 z}}{H_{0}^{2}} d z=\frac{1}{H_{0}^{2}} \int_{0}^{\infty} U_{0 z} H_{1 z} d z=-\frac{1}{H_{0}^{2}} \int_{0}^{\infty} U_{0} H_{1 z z} d z .
$$

$$
0=D H_{x x}-H+u^{r} \alpha^{-r}
$$

so that

$$
D H_{1 z z}(z) \sim-\alpha^{1-r} U_{0}^{r}(z)
$$

and

Finally,

$$
\int_{0}^{\infty} \frac{\Phi_{0} U_{0} H_{1 z}}{H_{0}^{2}} d z \sim \frac{\alpha^{1-r}}{D H_{0}^{2}} \int_{0}^{\infty} U_{0}^{r+1} d z
$$

$$
2 \int_{0}^{\infty} \frac{\Phi_{0} U_{0 z}}{H_{0}} d z=\frac{2}{H_{0}} \int_{0}^{\infty} \frac{U_{0 z}^{2}}{U_{0}} d z=\frac{2}{3} \eta^{3 / 2} \int_{0}^{\infty} \frac{\left(w_{y}(y)\right)^{2}}{w(y)} d y=\frac{2}{3} \eta^{3 / 2} .
$$

In summary, we obtain

$$
I_{1}=\sqrt{\eta}-\frac{1}{3} \eta^{3 / 2}+\frac{\alpha^{1-r}}{D H_{0}^{2}} \int_{0}^{\infty} U_{0}^{r+1} d z .
$$

Now

$$
I_{2}=\int_{0}^{\infty} \frac{U_{0} \Phi_{0}^{2}}{H_{0}} d z=\frac{1}{3} \eta^{3 / 2}
$$

and finally, we write

$$
I_{3}=\int U_{0} \frac{\Psi_{0 z}}{H_{0}^{2}}
$$

Now we have

$$
\frac{D \Psi_{0 z z}}{\alpha^{2}}-\Psi_{0}+r \alpha^{-r-1} U_{0}^{r} \frac{U_{0 z}}{U_{0}}=0
$$

so that

$$
\begin{aligned}
\Psi_{0 z} & \sim-\frac{\alpha^{1-r}}{D} U_{0}^{r} \\
I_{3} & \sim-\frac{\alpha^{1-r}}{D H_{0}^{2}} \int_{-\infty}^{\infty} U_{0}^{r+1} d z .
\end{aligned}
$$


Therefore, we finally obtain

$$
\operatorname{rhs}(55)=\alpha \sqrt{\eta}
$$

Combining this result with (56) and recalling that $\eta=\tanh ^{2} L$ (Proposition 1) yields (52). Note that $\left.\operatorname{lhs}(52)\right|_{\lambda=0}=\tanh ^{2} L<1$; on the other hand $\operatorname{lhs}(52) \rightarrow \infty$ as $\lambda \rightarrow \infty$. This shows that (52) admits a positive eigenvalue.

We now verify Lemma 6, by solving the full eigenvalue problem (19a, 19b, 21) numerically. The numerical algorithm consists of re-formulating the eigenvalue problem as a boundary value problem, by adjoining an extra equation $\frac{d}{d x} \lambda(x)=0$ along with an extra boundary condition $\phi_{x}(0)=1$. The inner approximation (54) was used as an initial guess. We then compare the resulting $\lambda_{\text {numeric }}$ with $\lambda_{\text {asymptotic }}$ obtained by solving numerically the algebraic equation (52). Using $r=2, D=1, L=1$ and with $p=90$ or $p=180$ we obtain:

$$
\begin{aligned}
& p=90: \quad \lambda_{\text {numeric }}=1.21197, \quad \lambda_{\text {asymptotic }}=1.13769 ; \quad \text { error }=6.5 \% \\
& p=180: \quad \lambda_{\text {numeric }}=1.1729, \quad \lambda_{\text {asymptotic }}=1.13769 ; \quad \text { error }=3.4 \% \text {. }
\end{aligned}
$$

It is clear that doubling $p$ halves the error. This provides a good numerical verification of Lemma 6 .

\section{Discussion}

In this paper we have studied the Gierer-Meinhardt system with large reaction rates. The main result, Theorem 2, is the classification of the stability of interior and boundary spike solutions. The behavior of the system differs significantly from the "standard" GM system (1). In particular, an interior spike is unstable with respect to translation instabilities, and moves towards the boundary. This is similar to the shadow GM system [18]. On the other hand, the interior spike of the standard GM system is stable [18], [39]. Therefore we expect that as the nonlinearity strength $p$ is decreased, the interior spike can be stabilized. It is an open question to determine this instability threshold.

In Theorem 3 we proved the stability of the large eigenvalue for a single spike under the assumption that $r=2$. We also conjecture that the theorem remains true for any $r>1$. It is an open question to prove this conjecture.

We have proved that large reaction rates are able to create spiky patterns in a similar way as has been shown before for small diffusion constant of the activator. In this sense, large reaction rates for the system increase its potential for pattern formation, even if the two diffusion constants are almost the same. This effect corresponds well to results in [15] where it is shown that Turing instability is possible for large reaction rates, even if the diffusion constants are almost the same.

Biologically, this is important, as it widens the range of possible applications for Turing systems to explain pattern formation into areas where there is no good justification for vastly different reaction rates but it 
is known that there are large reaction rates. If there is a high degree of cooperativity, which is typically the case for many gene hierarchies, a large reaction rate can often be explained theoretically and measured experimentally, thus opening the door for suitable Turing systems to explain the patterns observed.

\section{ACKnowledgements}

The work of JW is supported by an Earmarked Grant of RGC of Hong Kong. TK is supported by an NSERC discovery grant, Canada. MW and TK would like to thank the Department of Mathematics at CUHK for their kind hospitality, where a part of this paper was written.

\section{REFERENCES}

[1] P. W. Bates and P.C. Fife, The dynamics of nucleation for the Cahn-Hilliard equation, SIAM J. Appl. Math. 53 (1993), 990-1008.

[2] P. W. Bates and G. Fusco, Equilibria with many nuclei for the Cahn-Hilliard equation, J. Differential Equations 162 (2000), 283-356.

[3] P. W. Bates, E.N. Dancer, and J. Shi, Multi-spike stationary solutions of the Cahn-Hilliard equation in higher-dimension and instability, Adv. Differential Equations 4 (1999), 1-69.

[4] M. del Pino, P. Felmer, M. Kowalczyk Boundary Spikes in the Gierer-Meinhardt System, Commun. Pure Appl. Anal. 1 (2002), 137-156

[5] X. Chen and M. Kowalczyk, Slow dynamics of interior spikes in the shadow Gierer-Meinhardt system, Adv. Differential Equations 6 (2001), 847-872.

[6] X. Chen and M. Kowalczyk, Dynamics of an interior spike in the Gierer-Meinhardt system, SIAM J. Math. Anal. 33 (2001), 172-193.

[7] C. Gui and J. Wei, Multiple interior peak solutions for some singular perturbation problems, J. Differential Equations 158 (1999), 1-27.

[8] C. Gui, J. Wei and M. Winter, Multiple boundary peak solutions for some singularly perturbed Neumann problems, Ann. Inst. H. Poincaré Anal. Non Linéaire 17 (2000), 47-82.

[9] M. del Pino, M. Kowalczyk, and X. Chen, The Gierer-Meinhardt system: the breaking of homoclinics and multi-bump ground states, Commun. Contemp. Math. 3 (2001), 419-439.

[10] M. del Pino, M. Kowalczyk, and J. Wei, Multi-bump Ground States of the Gierer-Meinhardt system in $\mathbb{R}^{2}$, Ann. Non linearie, Annales de l'Institut H. Poincare 20 (2003), 53-85.

[11] A. Doelman, R. A. Gardner, and T. J. Kaper, Stability analysis of singular patterns in the 1D Gray-Scott model: a matched asymptotics approach, Phys. D 122 (1998), 1-36.

[12] A. Doelman, R. A. Gardner, and T. J. Kaper Large stable pulse solutions in reaction-diffusion equations, Indiana Univ. Math. J. 50 (2001), 443-507.

[13] A. Doelman, T. J. Kaper, and H. van der Ploeg, Spatially periodic and aperiodic multi-pulse patterns in the one-dimensional Gierer-Meinhardt equation, Methods Appl. Anal. 8 (2001), 387-414.

[14] A. Gierer and H. Meinhardt, A theory of biological pattern formation, Kybernetik (Berlin) 12 (1972), 30-39.

[15] A. Hunding and R. Engelhardt, Early biological morphogenesis and nonlinear dynamics, J. Theor. Biol. 173 (1995), 401413.

[16] P. W. Ingham, The molecular genetics of embryonic pattern formation in Drosophila, Nature 335 (1988), 25-34.

[17] D. Iron and M. J. Ward, The dynamics of boundary spikes for a non-local reaction diffusion model, Eur. J. Appl. Math. $11(2000), 491-514$

[18] D. Iron, M. J. Ward, and J. Wei, The stability of spike solutions to the one-dimensional Gierer-Meinhardt model, Phys. D $50(2001), 25-62$.

[19] D. Iron and M. J. Ward, The dynamics of multi-spike solutions to the one-dimensional Gierer-Meinhardt model, SIAM J. Appl. Math. 62 (2002), 1924-1951.

[20] T. Kolokolnikov and M. J. Ward, Reduced wave Green's functions and their effect on the dynamics of a spike for the Gierer-Meinhardt model, Eur. J. Appl. Math. 14 (2003), 513-545.

[21] H. Meinhardt, Models of biological pattern formation, Academic Press, London, 1982.

[22] H. Meinhardt, The algorithmic beauty of sea shells, Springer, Berlin, Heidelberg, 2nd edition, 1998.

[23] J. Murray, Mathematical Biology, II: Spatial models and Biomedical Applications, 3rd Edition, Springer-Verlag.

[24] W.-M. Ni, Diffusion, cross-diffusion, and their spike-layer steady states, Notices of Amer. Math. Soc. 45 (1998), 9-18.

[25] W.-M. Ni and I. Takagi, On the shape of least energy solution to a semilinear Neumann problem, Comm. Pure Appl. Math. 41 (1991), 819-851.

[26] W.-M. Ni and I. Takagi, Locating the peaks of least energy solutions to a semilinear Neumann problem, Duke Math. J. 70 (1993), 247-281.

[27] C. Nüsslein-Volhard, Determination of the embryonic axes of Drosophila, Development (Suppl.) 1 (1991), 1-10. 
[28] M. J. Pankratz and H. Jäckle, Making stripes in the Drosophila embryo, Trends Genet. 6 (1990), $287-292$.

[29] G. Riddihough, Homing in on the homeobox, Nature 357 (1992), 643-644.

[30] L. J. Slater, Confluent Hypergeometric Functions, Cambridge University Press, 1960.

[31] I. Takagi, Point-condensation for a reaction-diffusion system, J. Differential Equations 61 (1986), 208-249.

[32] A. M. Turing, The chemical basis of morphogenesis, Phil. Trans. Roy. Soc. Lond. B 237 (1952), 37-72.

[33] M. J. Ward et. al., The Dynamics and Pinning of a Spike for a Reaction-Diffusion System, SIAM J. Appl. Math. 62 (2002), $297-1328$.

[34] M. J. Ward and J. Wei, Asymmetric spike patterns for the one-dimensional Gierer-Meinhardt model: equilibria and stability, Eur. J. Appl. Math. 13 (2002), 283-320.

[35] M. J. Ward and J. Wei, Hopf bifurcation of spike solutions for the shadow Gierer-Meinhardt system, Eur. J. Appl. Math. 14 (2003) 677-711.

[36] J. Wei, On the boundary spike layer solutions of singularly perturbed semilinear Neumann problem, J. Diffential Equations 134 (1997), 104-133.

[37] J. Wei, On the interior spike layer solutions of singularly perturbed semilinear Neumann problem, Tohoku Math. J. 50 (1998), 159-178.

[38] J. Wei, On the interior spike layer solutions for some singular perturbation problems, Proc. Royal Soc. Edinburgh, Section A (Mathematics) 128 (1998), 849-874.

[39] J. Wei, On single interior spike solutions of Gierer-Meinhardt system: uniqueness and spectrum estimates, Eur. J. Appl. Math. 10 (1999), 353-378.

[40] J. Wei and M. Winter, On the two-dimensional Gierer-Meinhardt system with strong coupling, SIAM J. Math. Anal. 30 (1999), 1241-1263.

[41] J. Wei and M. Winter, Spikes for the two-dimensional Gierer-Meinhardt system: The weak coupling case, J. Nonlinear Science 6 (2001), 415-458.

[42] J. Wei and M. Winter, Spikes for the two-dimensional Gierer-Meinhardt system: The strong coupling case, J. Differential Equations 178 (2002), 478-518.

[43] J. Wei and M. Winter, Existence, classification and stablity analyisis of multiple-peaked solutions for the Gierer-Meinhardt system in $R^{1}$, Methods Appl. Anal. 14 (2007), 119-164.

[44] J. Wei and L. Zhang, On a nonlocal eigenvalue problem, Ann. Scuola Norm. Sup. Pisa Cl. Sci. 30 (2001), 41-61.

[45] D. G. Wilkinson, Molecular mechanisms of segmental patterning in the vertebrate hindbrain and neural crest, BioEssays 15 (1993), 499-505.

Department of Mathematics and Statistics, Dalhousie University, Halifax, Canada

E-mail address: tkolokol@mathstat.dal.ca

Department of Mathematics, The Chinese University of Hong Kong, Shatin, Hong Kong

E-mail address: wei@math.cuhk.edu.hk

Department of Mathematical Sciences, Brunel University, Uxbridge UB8 3PH, United Kingdom

E-mail address: matthias.winter@brunel.ac.uk, phone +441895267179 , fax +441895269732 , corresponding author 